\title{
SOBRE UMA NOVA ESPÉCIE NEOTRÓPICA DO GÊNERO Larsia FITTKAU, 1962 (DIPTERA: CHIRONOMIDAE: TANYPODINAE)
}

\author{
Arlindo Serpa-Filho
}

\begin{abstract}
On a new neotropical species of the genus Larsia Fittkau, 1962 (Diptera: Chironomidae: Tanypodinae) - This work presents the description of a new of the genus Larsia Fittkau, 1962 (Diptera: Chironomidae: Tanypodinae). The genus until the moment it is represented by 23 species in the world. In the Neotropical region, according to Spies \& Reiss (1996) four species were already described, being three from Guatemala, L. fittkaui Sublette \& Sasa, 1994, L. planensis Johannsen, 1946, L. reissi Sublette \& Sasa, 1994 and only from Argentina, L. pallescens Edwards, 1931. The new species was collected in the State of Rio de Janeiro, coming from Araras, municipal district of Petrópolis, RJ, being the first registration of the genus from Brazil. The slides with the material type are deposited in the Entomological Collection of the Instituto Oswaldo Cruz.
\end{abstract}

Keys words: Tanypodinae, Larsia, new species, Rio de Janeiro, Brazil

\section{Resumo}

Este trabalho apresenta a descrição de uma nova do gênero Larsia Fittkau, 1962 (Diptera: Chironomidae: Tanypodinae). O gênero até o

Departamento de Entomologia, Instituto Oswaldo Cruz/FIOCRUZ, Av. Brasil 4365, CEP: 21.040-900 Manguinhos, Rio de Janeiro, RJ, Brasil. E-mail: serpa@ioc.fiocruz.br 
momento está representado por 23 espécies no mundo. Na região Neotrópica, segundo Spies \& Reiss (1996) já foram assinaladas quatro espécies, sendo três na Guatemala, L. fittkaui Sublette \& Sasa, 1994, L. planensis Johannsen, 1946, L. reissi Sublette \& Sasa, 1994 e uma na Argentina, L. pallescens Edwards, 1931. A espécie nova foi coletada no Estado do Rio de Janeiro, procedente de Araras, município Petrópolis, RJ, sendo o primeiro registro do gênero no Brasil. Os exemplares testemunhos estão depositados na Coleção Entomológica do Instituto Oswaldo Cruz.

Palavras - chave: Tanypodinae, Larsia, espécie nova, Rio de Janeiro, Brasil

\section{Introdução}

Ao realizar revisão da subfamília Tanypodinae Fittkau (1962) descreveu o gênero Larsia, caracterizando o gênero pelas nervuras da asa, pelos esporões tibiais em forma de lira e pelas compridas cerdas que estavam presentes no gonocoxito. A ocorrência deste gênero se deu inicialmente pela descrição de uma espécie na Argentina, que recebeu a designação inicial de Pentaneura pallescens Johannsen, 1946 e que foi também assinalada no Chile por Brundin em 1966.

Morfologicamente são de tamanho pequeno apresentando na fase adulta características distintas como a presença de tubérculo escutal e esporões tibiais em forma de lira bem definidos.

Este gênero é muito comum em remanso e cabeceira de rios e igarapés que apresentem sedimento arenoso, mas podem ser encontrados em ambiente alterados por compostos orgânicos e poluentes.

Larsia é muito freqüente na região Neotrópica, porém até o momento está representado somente por quatro espécies. Segundo Spies e Reiss (1996), na Guatemala, foram descritas L. fittkaui Sublette \& Sasa, 1994, L. reissi Sublette \& Sasa, 1994, L. planensis (Johannsen, 1946) e na Argentina, L. pallescens (Edwards, 1931).

Neste trabalho descreve-se uma espécie nova do gênero Larsia Fittkau, 1962. A espécie nova, Larsia labartheae, é procedente de Araras, município de Petrópolis, RJ, onde deve-se ressaltar que esta é a primeira vez que se descreve uma espécie de adulto do gênero Larsia no Brasil. 


\section{Material e Métodos}

O material utilizado pertence ao acervo da Coleção Entomológica do Instituto Oswaldo Cruz e encontra-se preservado em álcool $70 \%$. As lâminas foram montadas com Euparal, segundo Schlee (1966). Todas as mensurações seguiram Epler (1988) e a terminologia morfológica e abreviações basearam-se em Saether (1980).

\section{Larsia labartheae sp. $\mathbf{n}$.}

(Figs. 1-3)

Macho (Figs. 1-2) - Tamanho: 2,8 mm ( $\mathrm{n}=3)$.

Cabeça (Fig.1a): Castanho-clara; antena com treze flagelômeros e todos fortemente plumosos e castanhos; tentório conforme figura $1 \mathrm{~b}$; cerdas temporais não ultrapassando ao número de vinte e oito e posicionadas em uma única fileira; olhos reniformes distância interocular de 0,36 mm; rebordo ocular moderadamente recoberto de cerdas e coloração castanho-claro; clípeo castanho-claro com 0,20 mm de comprimento, proeminente e recoberto por treze cerdas com coloração castanho-clara; palpômeros medindo em milímetros: 0,06: 0,10: 0,11: 0,09 (Fig. 1C). $A R=1,75$.

Tórax: com 0,76 mm de comprimento e cor castanha; área escutelar castanha; posnoto castanho; pleuras castanhas; quantidade de cerdas no tórax: 23 dorsocentrais dispostas em duas fileiras, 12 acrosticais, 13 prealares, 1 supralar, escutelares 16 ( 7 anteriores e 9 posteriores). Asa (Figs. 1d-e): com 1,77 mm de comprimento e 0,42 mm de largura; hialina e apresentando macrotríquias; $M$ robusta na parte basal e fina na parte apical; haste de Fcu posicionando-se junto a forquilha antes de Mcu; Mcu robusta e em oposição a r-m e, levemente mais acastanhada que as outras nervuras; franjas castanhas; squama com dezenove franjas amarelas; lobo anal pouco desenvolvido. $\mathrm{VR}=0,82$. Pernas: Todos os segmentos tibiais possuem cerdas medindo 0,10 $0,16 \mathrm{~mm}$. Esporões amarronzados e em forma de lira. Dentes dos esporões tibiais: $P_{1}: 10 ; P_{2}: 7 / 8 ; P_{3}: 6 / 7$ e pente com sete robustas cerdas (Fig. 2a-c). 


\begin{tabular}{llllllll||lll}
\hline & $\mathbf{f e}$ & $\mathbf{t i}$ & $\mathbf{t a}_{\mathbf{1}}$ & $\mathbf{T a}_{\mathbf{2}}$ & $\mathbf{T a}_{\mathbf{3}}$ & $\mathbf{T a}_{\mathbf{4}}$ & $\mathbf{T a}_{\mathbf{5}}$ & $\mathbf{L R}$ & $\mathbf{B V}$ & $\mathbf{S V}$ \\
\hline $\mathbf{P}_{\mathbf{1}}$ & 0,72 & 0,84 & 0,79 & 0,43 & 0,29 & 0,18 & 0,09 & 0,93 & 0,92 & 0,82 \\
$\mathbf{P}_{\mathbf{2}}$ & 0,78 & 0,71 & 0,65 & 0,29 & 0,18 & 0,12 & 0,09 & 2,37 & 3,14 & 2,82 \\
$\mathbf{P}_{\mathbf{3}}$ & 0,69 & 0,98 & 0,81 & 0,41 & 0,25 & 0,12 & 0,10 & 1,96 & 2.28 & 2,06 \\
\hline
\end{tabular}

Abdome (Fig. 2d): não ultrapassando 1,71 mm de comprimento; segmentos abdominais castanho-claros, revestidos de cerdas castanho-escuras que recobrem o tergito e o esternito; todos os segmentos abdominais apresentam uma faixa, castanho-escura, que se alarga nos três últimos segmentos.

Hipopígio (Fig. 2e): castanho-claro com gonocoxito robusto e levemente sub-cônico com longas cerdas que recobrem toda a genitália, não ultrapassando ao número de quarenta e cinco cerdas; comprimento de $0,13 \mathrm{~mm}$ de e largura de $0,07 \mathrm{~mm}$; gonostilo com 0,11 mm de comprimento, não ultrapassando de $2 / 3$ do comprimento do gonocoxito, megasseta de cor castanho-escura.

Fêmea (Fig. 3): Tamanho: 1,8 mm ( $\mathrm{n}=3)$.

Cabeça (Fig.3b): castanho-clara; antena com onze flagelômeros, dez cerdas temporais dispostas em uma única fileira; distância inter-ocular igual a 0,29mm; rebordo ocular com algumas cerdas; clípeo castanho, com $0,23 \mathrm{~mm}$ de comprimento e recoberto de cerdas que variam de 27 34 e com coloração castanho-clara; palpômeros medindo respectivamente em milímetros: 0,07: 0,12: 0,18: 0,18 (Fig. 3c).

Tórax $(n=1)$ : castanho-escuro, com $0,80 \mathrm{~mm}$ de comprimento; pronoto castanho-escuro; mesonoto castanho-escura; área escutelar castanha; posnoto castanho-escuro; pleuras castanhas; quantidade de cerdas no tórax: 36 dorsocentrais dispostas em duas fileiras, 25 acrosticais, 10 prealares, 1 supralar, escutelares 16 (10 anteriores e 14 posteriores).

Asa (Fig. 3a): com 1,7 mm de comprimento; hialina, com poucas microtríquias; haste de Fcu posicionando-se junto a forquilha em cima de Mcu; Mcu robusta e em oposição a r-m; r-m e Mcu levemente mais acastanhadas que as outras nervuras; franjas castanhas; squama não ultrapassando de vinte e cinco franjas amarelas; lobo anal pouco desenvolvido. $\mathrm{VR}=0,85$.

Pernas: Todos os segmentos tibiais possuem cerdas medindo 0,10 - 0,16 $\mathrm{mm}$; esporões tibiais da cor castanho-escura e em forma de lira. Dentes dos esporões tibiais: $\mathrm{P}_{1}: 7 ; \mathrm{P}_{2}: 8 / 9 ; \mathrm{P}_{3}: 7 / 9$ e pente com 7 cerdas. 


\begin{tabular}{llllllll||lll}
\hline & $\mathbf{f e}$ & $\mathbf{t i}$ & $\mathbf{t a}_{\mathbf{1}}$ & $\mathbf{T a}_{\mathbf{2}}$ & $\mathbf{T a}_{\mathbf{3}}$ & $\mathbf{T a}_{\mathbf{4}}$ & $\mathbf{T a}_{\mathbf{5}}$ & $\mathbf{L R}$ & $\mathbf{B V}$ & $\mathbf{S V}$ \\
\hline $\mathbf{P}_{\mathbf{1}}$ & 0,68 & 0,92 & 0,76 & 0,40 & 0,29 & 0,16 & 0,10 & 0,82 & 2,49 & 2,09 \\
$\mathbf{P}_{\mathbf{2}}$ & 0,80 & 0,92 & 0,70 & 0,35 & 0,32 & 0,16 & 0,10 & 0,76 & 2,88 & 2,45 \\
$\mathbf{P}_{\mathbf{3 ( n = 2 )}}$ & $\mathbf{0 , 7 7}$ & $\mathbf{1 , 0 5}$ & $\mathbf{0 , 6 7}$ & $\mathbf{0 , 3 2}$ & $\mathbf{0 , 2 2}$ & $\mathbf{0 , 1 5}$ & 0,10 & 0,63 & 3,22 & 2,72 \\
\hline
\end{tabular}

Abdome $(n=1)$ : não ultrapassando de $1,0 \mathrm{~mm}$; segmentos abdominais castanhos-escuros, revestidos de cerdas castanhas que recobrem o tergito e o esternito.

Genitália (Fig. 3d): não muito diferentes das outras espécies do gênero; lobo dorsomesal da gonapófise VIII é curto e grosso; dutos espermáticos fino e bem longos; cápsulas seminais com coloração acastanhada e formato oval (Fig. 3e); X segmento sem cerda; cercus medindo 0,08mm; coxosternapodema em forma de forquilha invertida, gonocoxito reduzido e um pouco achatado; outras estruturas não puderam ser observadas devido a montagem das lâminas.

Larva e pupa: desconhecidas.

Material examinado: Holótipo macho, BRASIL, Rio de Janeiro, Petrópolis, Araras, Sítio Querência, 09/12/1995, N. Labarthe col.. Alótipo fêmea, coletado junto com o macho. Parátipos: 1 macho e 2 fêmeas coletados com o holótipo. Depositados na Coleção Entomológica do Instituto Oswaldo Cruz.

Etimologia: designado em homenagem a Dra. Norma Volmer Labarthe.

\section{Discussão}

Macho: Larsia labartheae sp. n. se aproxima de L. reissi pelo número de dentes tibiais, mas se diferencia facilmente de L. planensis, L. reissi, L. pallescens, L. fittkaui pelos maiores valores: 0,93, 0,92 e 0,82 da LR em $\mathrm{P}_{1}, \mathrm{P}_{2}$ e $\mathrm{P}_{3}$, respectivamente. Quanto a VR de L. labartheae apresentando 0,82 , este menor que em relação a $L$. reissi, $L$. fittkaui. $A$ AR igual a 1.75, apresenta-se maior quando comparados às espécies já citadas acima. As proporções dos segmentos do palpo são ligeiramente menores que os das outras espécies neotrópicas, além de apresentar o comprimento do último segmento menor que o terceiro. Para a medida da asa, notou-se maior que nas outras espécies 

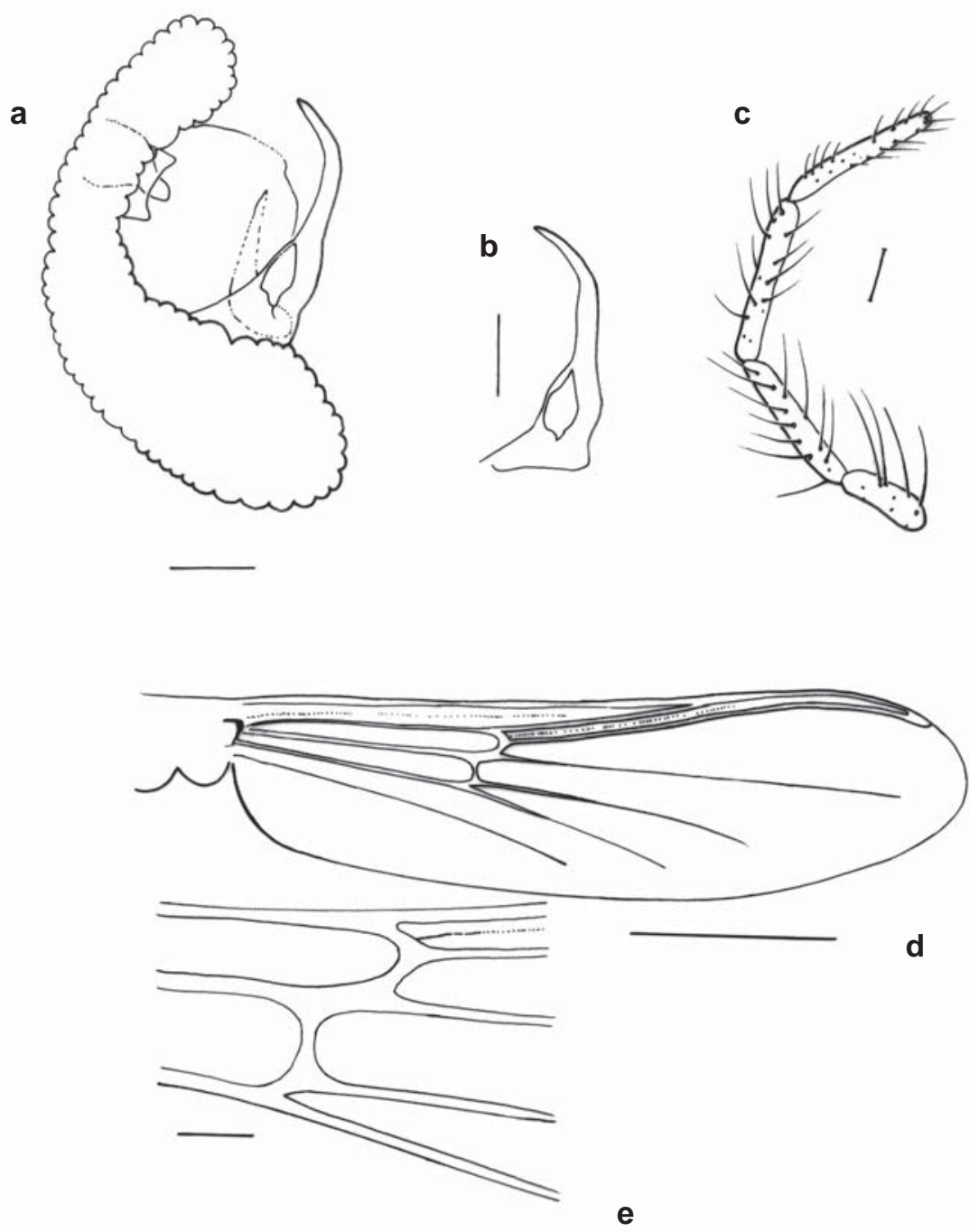

Figura 1: Larsia labartheae sp. n., macho. a: detalhe da cabeça; b: tentório; c: palpo; d: asa; e: detalhe das nervuras r-m e m-cu. 
a

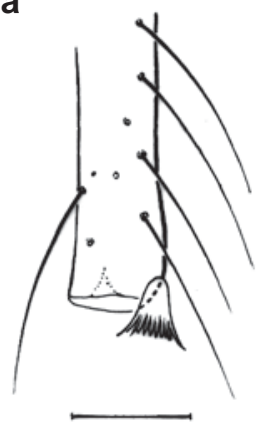

d

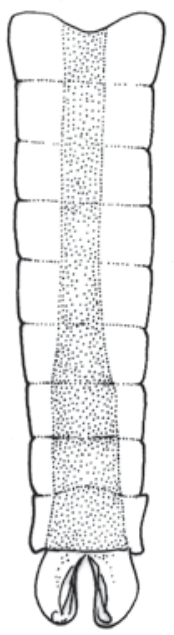

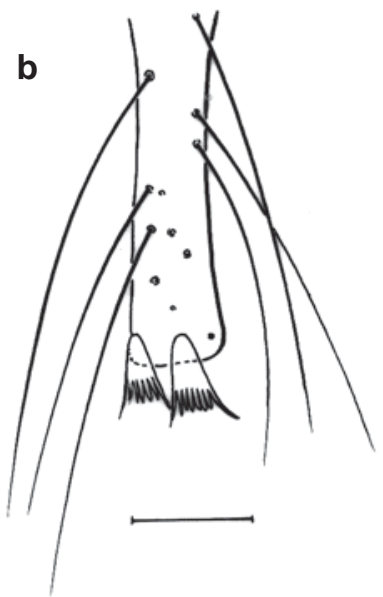

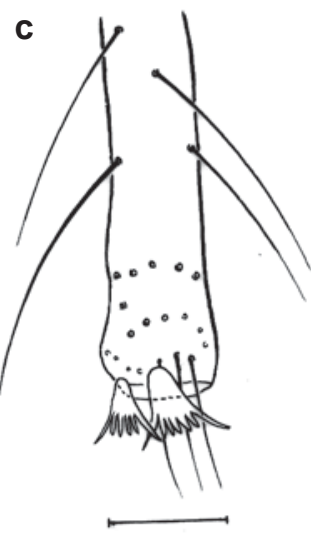

e

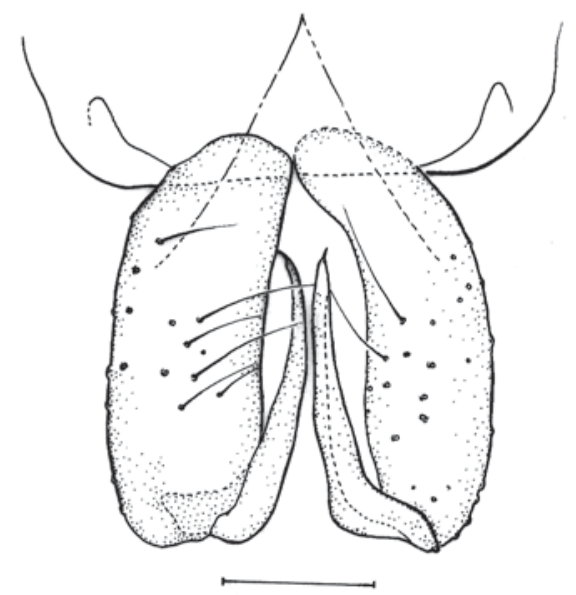

Figura 2: Larsia labartheae sp. n., macho. a: tíbia anterior; b: tíbia média; c: tíbia posterior; d: desenho esquemático da região dorsal dos segmentos abdominais; e: genitália. 

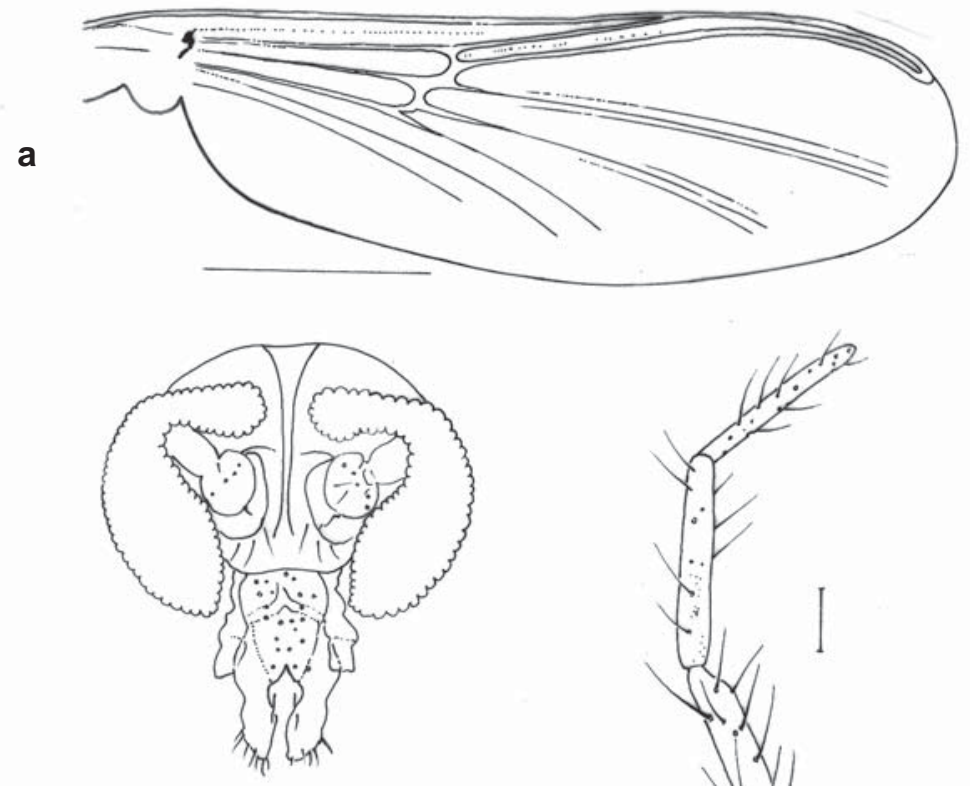

b
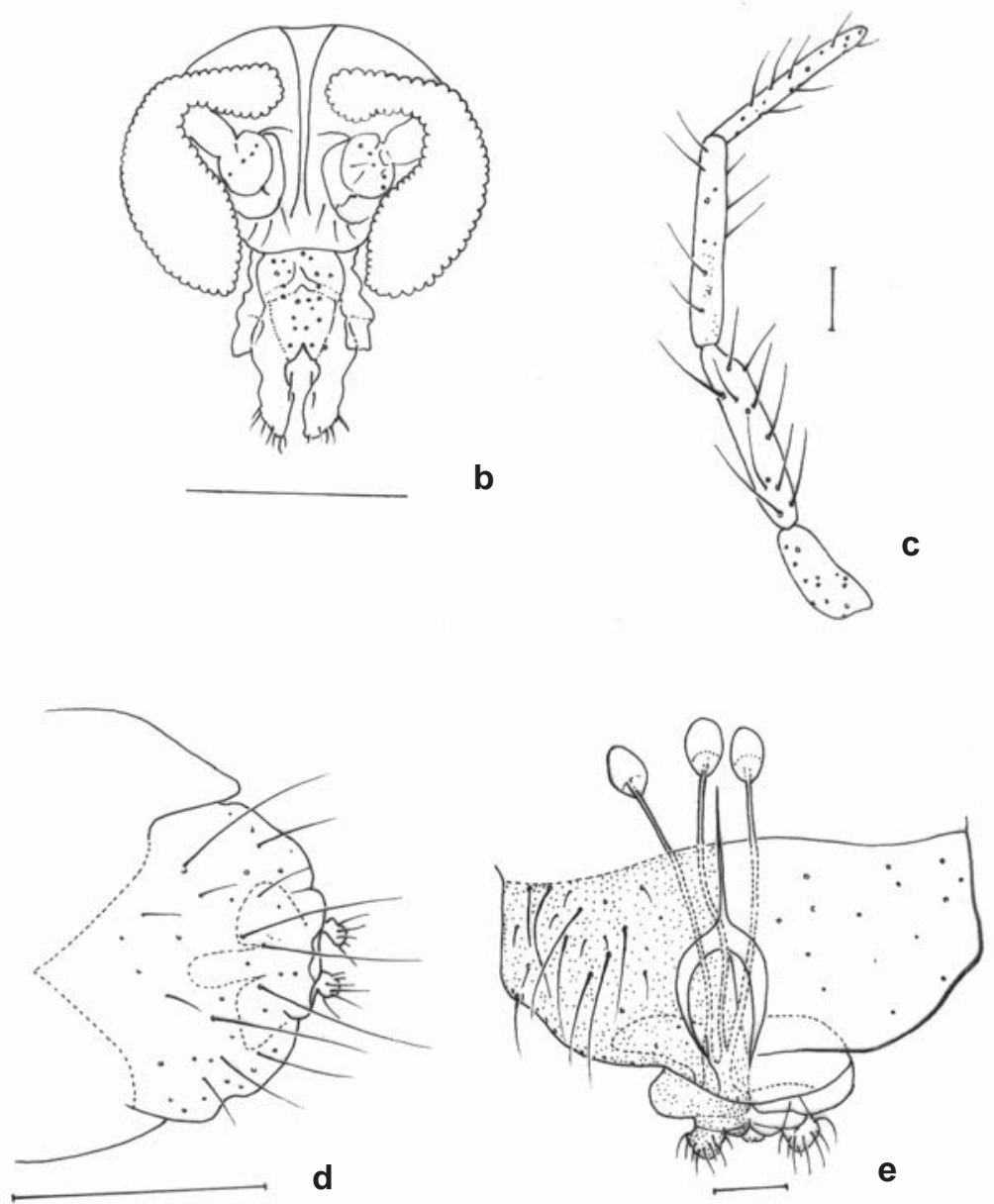

Figura 3: Larsia labartheae sp. n., fêmea. a: asa; b: cabeça; c: palpo; d: vista lateral da genitália; e: genitália com detalhe das cápsulas seminais. 
neotrópicas tanto nos machos como nas fêmeas. A coloração da asa só diferiu em relação à espécie L. planensis, principalmente na cor das nervuras da asa. As características da genitália revelaram que a mesma difere das outras espécies já descritas, pelo formato oval e o número demasiado de cerdas longas e curtas.

Fêmea: Larsia labartheae sp. n. se aproxima das outras espécies neotrópicas, pelo número de dentes tibiais. Difere de $L$. reissi, L. fittkaui, e $L$. friedrichi pelas medidas maiores da asa. A VR igual a 0,85 de $L$. labartheae é menor que em $L$. reissi, $L$. fittkaui. Os valores de $L R$ de $P_{1}, P_{2}$ e $P_{3}$ nesta espécie são respectivamente $0,82,0,76,0,63$ e diferem em relação as espécies de $L$. reissi e L. fittkaui.

\section{Agradecimentos}

Ao Prof. Dr. Sebastião José de Oliveira (in memoriam), ex-Curador da Coleção Entomológica do Instituto Oswaldo Cruz, pelo aprendizado e pelas valiosas informações nos estudos sobre Chironomidae.

\section{Referências Bibliográficas}

Armitage, P.D.; Cranston, P.S. \& Pinder, L.C.V., 1995. The Chironomidae. Biology and ecology of non-biting midges. 1st Ed. London, Chapman \& Hall. 538 p.

Edwards, F.W., 1931. Chironomidae, p. 233-324 In: Diptera of Patagonia and South Chile II, volume 5.

Epler, J.H., 1988. Biosystematics of the genus Dicrotendipes Kieffer, 1913 (Diptera: Chironomidae) of the world. Mem. Am. Entomol. Soc. 36:1-214.

Fittkau, E.J., 1962. Die Tanypodinae (Diptera: Chironomidae). (Die tribus Anatopyniini, Macropelopiini und Pentaneurini). Abh. Larv. Syst. Insekt. 6:1-453. 
Johannsen, A.O., 1946. Revision of the North American species of the genus Pentaneura (Tendipedidae:Chironomidae:Diptera). J. N.Y. Soc 54:267289.

Reiss F., 1981. Chironomidae. p. 261-268, In: Aquatic biota of tropical South America. Part 1. Arthropoda. Hurlbert, SH, Rodriguez, G, Santos ND (eds).. San Diego State University, San Diego, California. 323 p.

Roback, S.S., 1971. The adults of the subfamily Tanypodinae (=Pelopiinae) in North America (Diptera: Chironomidae). Monog. Acad. Nat. Sci. Phil. 17.1-410p.

Roback, S.S., 1971. The adults of the subfamamily Tanypodinae (=Pelopiinae) in North America (Diptera: Chironomidae). Monograph 17. The Academy of Natural Sciences of Philadelphia 19 th and the Parkway Philadelphia, Pennsylvania 19103.

Saether, A.O., 1977. Female genitalia in Chironomidae and other Nematocera: morphology, phylogenies, keys. Dipt. Fish. Envir. Fish. Mar. Ser. 197:1-209.

Saether, A.O., 1980. Glossary of the chironomid morphology terminology (Diptera: Chironomidae). Entomol. Scand. 14 Suppl:1-31.

Schlee, D., 1966. Präparation und Ermittlung von Messwerten an Chironomidae (Diptera). Gewäss. Abwäss. 41/42:169-193.

Spies, M. \& Reiss, F., 1996. Catalog and bibliography of Neotropical and Mexican Chironomidae (Insecta, Diptera). Spixiana 22 Suppl: 61-119.

Sublette, J.E. \& Sasa, M., 1994. Chironomidae collected in Onchocerciasis endemic areas of Guatemala. Spixiana 20 Suppl: 1-60. 